\title{
Les Mondes de Labiche, dir. O. BARA, V. HEYRAUD et J.- Cl. YON
}

Lise Sabourin

\section{(2) OpenEdition \\ Journals}

Édition électronique

URL : https://journals.openedition.org/studifrancesi/32033

DOI : $10.4000 /$ studifrancesi.32033

ISSN : 2421-5856

\section{Éditeur}

Rosenberg \& Sellier

Édition imprimée

Date de publication : 1 août 2020

Pagination : 425-426

ISSN : 0039-2944

Référence électronique

Lise Sabourin, "Les Mondes de Labiche, dir. O. bara, V. heyraud et J.-Cl. yon », Studi Francesi [En ligne], 191 (LXIV | II) | 2020, mis en ligne le 01 septembre 2020, consulté le 18 septembre 2021. URL : http:// journals.openedition.org/studifrancesi/32033; DOI : https://doi.org/10.4000/studifrancesi.32033

Ce document a été généré automatiquement le 18 septembre 2021.

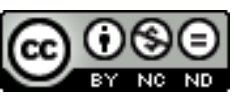

Studi Francesi è distribuita con Licenza Creative Commons Attribuzione - Non commerciale - Non opere derivate 4.0 Internazionale. 


\title{
Les Mondes de Labiche, dir. O. BARA, V. HEYRAUD et J.-Cl. YON
}

\author{
Lise Sabourin
}

\section{RÉFÉRENCE}

Les Mondes de Labiche, dir. O. BARA, V. HEYRAUD et J.-Cl. YON, Paris, Presses de la Sorbonne Nouvelle, 2017, 279 pp.

1 Labiche, quoique son nom reste attaché au répertoire léger $d u$ xix ${ }^{e}$ siècle, demeure méconnu, soit ravalé par sa pratique de l'écriture en collaboration, soit jugé trop mental pour son appréciation des déviances bourgeoises. Le colloque réuni par Olivier Bara, Violaine Heyraud et Jean-Claude Yon, à l'occasion du bicentenaire de sa naissance en 2015, s'organise en quatre parties: «Labiche dans le monde théâtral», «Le monde selon Labiche», «Labiche à travers le monde» et «Le monde après Labiche».

Dans la première section, Aline MARCHADIER étudie les Discours et écrits critiques de Labiche, véritable homme de plume doté d'une poétique théâtrale (pp.23-33). Il a des préoccupations esthétiques et morales, sait pratiquer une gaîté naturelle très lucide sur les défauts humains. Barbara T. COOPER se penche sur Labiche, auteur de drames (pp. 35-44): L'Avocat Loubet (théâtre du Panthéon, 28 août 1838), La Forge des châtaigniers (théâtre Saint-Marcel, 4 avril 1839), La Peine du talion ou les Représailles (théâtre du Luxembourg, 5 juin 1839) constituent un véritable laboratoire dramaturgique, assez proche du romantisme dumasien, avec des personnages bien saisis, un dialogue vif, des intrigues munies de péripéties, des scènes et bruits du quotidien. Mariglen sUlEjMANI discerne dans Quelques aspects matériels du théâtre de Labiche sur la scène du Palais-Royal (pp. 45-56) combien l'étroitesse de sa «petite salle» l'a obligé à concevoir sa proximité avec le public sur le mode de la farce au temps de ses débuts avec Marc-Michel, lui indiquant les jeux de physionomie des acteurs, le caractère convivial de la scène comme de puissants instruments pour son comique de caractère. Patrick BERTHIER voit aussi dans «l'odyssée frénétique» avec ses acteurs de Labiche jusqu'à “Un Chapeau de paille 
d'Italie" (pp. 57-68) une des raisons de l'accélération d'intrigues qui marquera l'œuvre de cet «adroit vaudevilliste». Agnès SANDRAS s'interroge à propos De la difficulté d'ironiser sur le maitre du rire. Les petits piques humoristiques de ses contemporains contre Labiche (pp. 69-81) s'expliquent aussi par ses traits ironiques de satiriste, en un jeu de réciprocité percutant, en réalité fort maîtrisé, qui va même jusqu'à faire rire ses académiques confrères lors de sa réception sous la Coupole.

Dans la deuxième partie, Sylvain NIColle s'interroge sur Labiche, candidat et vaudevilliste sous la II République: de l'engagement politique au désenchantement? (pp. 85-98): ce républicain romantique lyrique, après son échec électoral en 1848 , reste soucieux de méritocratie, mais s'indigne devant les effets du suffrage universel liés à l'ignorance populaire et à la tartuferie des clubs. Il développe de ce fait en 1848-49, avec Le Club champenois, L'Exposition des produits de la république et Rue de l'homme armé, 8 bis, un conservatisme qui le rendra finalement favorable à Napoléon III. Janice BEST étudie le hors-scène et la subversion des valeurs dans trois pièces de Labiche (pp. 98-111): que ce soit dans Une Fille bien gardée, Un Chapeau de paille d'Italie ou La Perle de la Canebière, la représentation du monde populaire constitue un espace de désordre subreptice qui en fait exploite les failles de la morale bourgeoise, rétablie apparemment mais présentée comme temporaire. Carine GOUTALAND se penche sur les mondes alimentaires de Labiche ("À table! à table vite! [...] l'appétit vaut mieux que l'esprit», pp. 113-123) en cette époque de développement du discours gastronomique, de l'hédonisme et d'affinement des codes sociaux. Elle montre comment Labiche utilise les lieux parisiens reconnus (Véry, Véfour, Le Bœuf à la mode, le traiteur Chevet, la Maison dorée, le Café anglais, le restaurant Brébant...), leurs mets et vins fins, mais aussi leurs recettes et leurs joutes culinaires, l'atmosphère des cabinets particuliers, dépeignant sans fard les transgressions de la société offenbachienne. Amina KHARROUBY récapitule la relation entre Eugène Labiche et la satire de la bourgeoisie vénale (pp. 124-136), tandis que Vincent GIROUD étudie le rapport de Labiche et l'Amérique (pp.137-147): pour «le philistin» français l'Américain, riche et lointain, relève d'un stéréotype caricatural fort comique. Ignacio RAMOS GAY décèle, avec le «sauvage» rastaquouère, héritier des conquistadors et des indiens à la fois, des reflets d'animalisation et darwinisme dans "Le Prix Martin" (pp. 149-160).

4 La troisième section s'élance d'abord dans La diffusion des pièces de Labiche en province et au Maghreb jusqu'à la première guerre mondiale par l'étude de Christine CARRÈRE-SAUCÈDE (pp. 163-172). Jouer «du Labiche» dans les grands comme les petits théâtres, en tournées d'acteurs comme par des amateurs en salons, fait toujours recette favorisant les inaugurations de salles, les ouvertures de saisons, les représentations à bénéfices d'artistes, puisque tous les publics sont touchés par sa verve. Michel RAPOPORT étudie $L a$ fortune britannique de Labiche (pp. 173-180): trente-trois de ses pièces sont représentées, surtout dans les années 1870-1890 et après 1950, soit en français, soit en adaptations anglaises, tandis que fleurissent aussi les éditions; sans doute le relatif manque d'œuvres britanniques relevant de la vis comica en fin de XIX ${ }^{e}$ siècle explique-t-il ce goût pour ses intrigues, dont la gauloiserie et l'extravagance séduisent le public. Giovanna BELLATI plaide Pour une introduction à la diffusion et à la fortune d'Eugène Labiche en Italie (pp. 181-190): dès les années 1850, quelques pièces traduites et jouées, outre celles représentées par les tournées françaises, séduisent le public de la péninsule, notamment La Cagnotte adaptée par Arrigui en 1860, puis par Fontana un siècle plus tard, ce qui prépare à la vogue des radiodramma des années 1950 puis aux adaptations 
télévisées. Konstanza GEORGAKAKI constate au contraire le manque de succès de Labiche sur la scène du théâtre national de Grèce (pp. 191-199) tant que les metteurs en scène n'ont pas pris la peine d'adapter costumes et mœurs aux us locaux, le comique labichien paraissant trop farcesque ou vulgaire au public athénien. Geneviève DE VIVEIROS en revanche constate le passage D'une scène à l'autre: Labiche au Canada au XIX siècle (pp. 201-211): avec un détour fréquent par leur traduction américaine, les pièces de Labiche reviennent au public francophone de Montréal qui apprécie leur représentation lors des fêtes collectives, des levées de fonds, des causes sociales à défendre, le public appréciant leur rythme endiablé, leurs effets comiques portés par de bons acteurs.

5 La quatrième partie s'ouvre sur la façon dont est porté Labiche, de la scène à l'écran (pp. 215-228): Valentina PONZETTo confronte les deux films de René Clair, Un Chapeau de paille d'Italie et Les Deux Timides à ceux de Maurice Cammage et d'Yves Allégret. Si le premier a su rendre spectaculaire le «vaudeville cauchemar», le passage au parlant a moins réussi paradoxalement à Labiche, dont les motifs proches du cinéma muet avaient fait florès (course-poursuite, jeune homme maladroit miraculeusement sauvé, gendarmes peu futés, quiproquos multiples et noce avec parfum d'adultère). Claudine GROSSIR montre Le théâtre de Labiche, aux marges de l'enseignement secondaire (pp. 229-238): les manuels ont eu du mal à insérer son œuvre dans les mouvements littéraires du XIXe, souvent limités théâtralement au romantisme ou au symbolisme; c'est seulement récemment avec l'introduction notamment du Voyage de M. Perrichon, d'Un Chapeau de paille d'Italie et, à moindre titre de La Grammaire et de L'Affaire de la rue de Lourcine que les collégiens ont pu découvrir des pièces entières de Labiche. Marion DENIzoT enfin souligne combien Eugène Labiche a pu constituer un objet illégitime pour les études théâtrales (pp. 239-250) à leur naissance dans les années 1980, son travail en collaboration, sa fonction divertissante ayant souvent brouillé l'image auctoriale alors que le succès public persistait.

6 Ce volume, enrichi d'un cahier d'illustrations, d'une bibliographie, d'index des noms propres et des pièces de Labiche, est vraiment intéressant et bien construit dans son évolution: il corrobore tout l'intérêt du travail d'édition en cours, auquel participent beaucoup des contributeurs, du Théâtre complet de Labiche, dont les éditions du XIX ${ }^{\mathrm{e}}$ siècle ne donnent que bien partiellement la vision. 